\title{
Identificação dos maiores desafios referentes à cultura e à comunicação organizacionais para a implantação de um sistema de gestão do conhecimento em uma companhia pública brasileira
}

\author{
Identification of the biggest challenges regarding organizational culture and communication \\ for the implementation of a knowledge management system in a brazilian public company
}

\author{
REZENDE, Terezinha C. ${ }^{1}$ \\ LEITE, Janaína P. ${ }^{2}$ \\ OLIVEIRA, Ana L.C. ${ }^{3}$
}

\section{Resumo}

Mudanças comportamentais são pré-requisitos à gestão do conhecimento. A literatura privilegia aspectos tecnológicos, mas existem desafios à sua implementação referentes à cultura e à comunicação. Objetivou-se identificar os maiores desafios na visão de gestores de recursos humanos, comunicação e tecnologia da informação de uma companhia pública brasileira. Análises das entrevistas identificou como obstáculos: conservadorismo, hierarquia rígida, isolamento da diretoria, conflito de gerações enquanto engajamento do capital humano e corpo técnico robusto seriam facilitadores, desse processo vital às empresas.

Palavras-chave: conhecimento, cultura organizacional, comunicação organizacional, gestão do conhecimento.

\begin{abstract}
Behavioral changes are prerequisites to knowledge management. Literature emphasizes technological aspects, but there are challenges to its implementation related to culture and communication. The objective was to identify the biggest challenges in the vision of human resources, communication and information technology managers of a Brazilian public company. Analysis of the interviews identified as obstacles: conservatism, rigid hierarchy, isolation from the board, generational conflict while the engagement of human capital and robust technical staff would facilitate this vital process for companies. Keywords: knowledge, organizational culture, organizational communication, knowledge management.
\end{abstract}

\footnotetext{
${ }^{1}$ Centro Universitário UNA. Brasil. Mestre em Administração de Empresas. Email: terezasc.r@gmail.com

${ }^{2}$ Escola Superior de Propaganda e Marketing. MBA em Gestão de Comunicação em Mídia Digital. E-mail: janaina05@hotmail.com

${ }^{3}$ Universidade Federal de Minas Gerais. Doutora em Engenharia Civil. Email: anacrespo.oliveira@gmail.com
} 


\section{Introdução}

As organizações contemporâneas estão inseridas em um ambiente caracterizado, principalmente, pela alta competitividade, pela redução do ciclo de vida de produtos e pela integração dos mercados, no qual a informação e o conhecimento se tornaram essenciais para o desempenho das empresas (Toffler, 1990). Os tradicionais fatores de produção - terra, trabalho e capital - não desapareceram, mas tornaram-se secundários frente ao conhecimento (Drucker, 1995) reconhecido como o principal ativo das organizações e a chave para a vantagem competitiva sustentável (Davenport \& Prusak, 2003). A possibilidade de o conhecimento se tornar uma vantagem competitiva está mais relacionada à aplicação do conhecimento do que ao mercado (Xavier, Oliveira, \& Teixeira, 2012) abrindo-se espaço para a Gestão do Conhecimento que, para ter sucesso em um ambiente corporativo, exige um movimento organizacional que direcione ações nas dimensões de processos, pessoas e tecnologia (Contador, 2005). A Gestão do Conhecimento tem privilegiado os aspectos de processos e tecnologias e negligenciado as pessoas (Rossetti \& Morales, 2007). Contudo, sem a participação e o envolvimento dos funcionários, torna-se difícil pensar o êxito de uma mudança como a Gestão do Conhecimento exige. A cultura organizacional desempenha um papel capaz de influenciar, direta ou indiretamente, tanto o sucesso da implantação como o uso da tecnologia da informação (Fernandes, Alturas, \& Laureano, 2015) sendo importante a motivação de seus colaboradores para contribuírem com suas ideias (Sales, Ferreira, Paiva, \& Ziviani, 2019). A informação apresenta como função fundamental integrar propósitos, processos e pessoas nas organizações reservando-se à comunicação a tarefa de fazer com que esses elementos estejam alinhados à estratégia organizacional (Andrade \& Roseira, 2018). A essa função essencial reúnem-se outras, como auxiliar no desenvolvimento e no crescimento da organização e assegurar o comprometimento dos empregados (Paiva \& Alves, 2013). Deve-se reconhecer a importância da cultura e da comunicação organizacionais para um processo no qual pessoas precisam ser levadas em conta, assim como os hábitos, os medos, as resistências e as expectativas que carregam, sob pena de o sistema de gestão do conhecimento desenvolvido não se mostrar eficaz em termos práticos (Bhatt, 2002).

A pesquisa se justificou a partir de diferentes perspectivas do ponto de vista da companhia em estudo: identificar aspectos que podem potencializar a implantação de um sistema-piloto de gestão do conhecimento em determinada área da organização; discutir aspectos tanto para a eficiência das operações, quanto para a capacidade de inovação da empresa; evitar que o conhecimento se perca e, em contrapartida, aumentar a sua competitividade no mercado. $O$ objetivo da realização deste trabalho foi a identificação dos maiores desafios a serem enfrentados, referentes à cultura e à comunicação organizacionais, para a implantação de um sistemapiloto de gestão do conhecimento, na visão dos gestores de uma companhia pública brasileira.

\section{Referencial teórico}

\subsection{Conhecimento e Gestão do Conhecimento}

Para (Davenport \& Prusak, 2003), o conhecimento pode ser entendido como uma mistura fluida de experiência condensada, valores, informação contextual e insight experimentado, que proporciona uma estrutura para a avaliação e incorporação de novas práticas e informações. Nas organizações, costuma encontrar-se embutido em documentos e repositórios e, também, em rotinas, processos, práticas e normas organizacionais. Para (Nonaka \& Takeuchi, 1997) o conhecimento tácito é subjetivo, manifestado em contexto específico, associado à experiência de quem o detém sendo, portanto, difícil de sistematizar e comunicar enquanto o conhecimento explícito está relacionado à racionalidade, ao formal, ao sistemático sendo mais facilmente transmissível. Nonaka (1994), (Choo, 2003) e (Alvarenga Neto, 2005) corroboram ao visualizarem a importância da sinergia entre o 
conhecimento tácito e o conhecimento explícito dentro de uma organização. Segundo (Xavier, Oliveira, \& Teixeira, 2012), as empresas que privilegiam o conhecimento tácito buscam fomentar e desenvolver atividades ligadas ao maior compartilhamento de ideias e experiências, à maior frequência de contato e interação entre os funcionários da organização. Ideia compartilhada por (Dazzi \& Angeloni, 2009) que ressaltam que é preciso criar condições adequadas para o desenvolvimento do conhecimento, incentivando a integração entre os indivíduos, propiciando o diálogo e o relacionamento, criando um ambiente favorável para gerar o conhecimento. No entanto, (Runte \& OLiveira, 2012) lembram que o uso de sistemas para armazenar, processar e transformar os dados em informações é necessário para a codificação do conhecimento e o maior embasamento para a tomada de decisões estratégicas dos gestores da organização. Portanto, conforme afirmam (López \& Junquera, 2013), nas empresas que dão preferência ao conhecimento explícito, a gestão do conhecimento encontra-se focada nas tecnologias para armazenagem, codificação e reaproveitamento do conhecimento enquanto naquelas que focam as experiências compartilhadas caracterizam-se como a transferência de conhecimento organizacional, que conferem melhorias nos processos e nos resultados. Na percepção de (Moreno \& Santos, 2012), a Gestão do Conhecimento constitui, com toda sua multidisciplinariedade e variáveis, um processo que integra, na estratégia organizacional, a gestão de pessoas, tecnologias de informação e comunicação. Nesse contexto, (Silveira , Ziviani, Ferreira, \& Paiva, 2018) reconhecem que a transferência do conhecimento tácito seja um fator relevante para as organizações por ser fonte de vantagem competitiva organizacional.

\subsection{Cultura e Comunicação organizacionais}

A cultura organizacional é sinalizada como um fator crítico de sucesso no processo de Gestão do Conhecimento (Cruz \& Ferreira, 2015) cujos principais seriam os valores, crenças, rituais, estórias e mitos, tabus, heróis, normas e processos de comunicação (Freitas, 2006). (Schein, 2004) descreve três tipos de culturas que se alternam nas organizações: a coercitiva (os empregados devem obedecer a quaisquer regras que lhes forem impostas); a utilitária (ambiente em que as regras são essenciais, porém, os membros protegem-se por meio de questionamentos e rejeições a certos valores ou práticas da empresa) e a normativa (existe uma equalização entre os objetivos da empresa e os do indivíduo), entre as quais coexistem diferentes dimensões de autoridade (autocráticas, paternalistas, democráticas, participativas; de delegação e abdicadoras - que concedem também poder e controle). No entanto, o processo de mudança organizacional é inerente à vida das organizações e é condição para a adaptação, sobrevivência e efetividade delas (Silva \& Vergara, 2003); (Wood Júnior, T., 2009); (Neves \& Castro, 2010). (Carvalho, 2012) ressalta que as mudanças, no decorrer dos anos, acarretaram impactos no ambiente de trabalho, como o vínculo com a cultura organizacional, a relação entre liderança e subordinados, a construção de laços sociais, assim como o reconhecimento profissional. A cultura cumpre a função de fornecer estabilidade, significado e previsibilidade no presente, mas é o resultado de decisões funcionalmente eficazes no passado do grupo (Schein \& Schein, 2017) sendo fator dominante de sucesso no processo de implantação da gestão do conhecimento (Zandi, Moghadam, \& Ali Pour, 2016). Para (Perides, Vilela, Souza, Dutra, \& Casado, 2020), o modelo de gestão de pessoas por competências no processo de transformação da cultura organizacional é relevante desde que as competências individuais definidas e valorizadas pela organização estejam alinhadas com as competências organizacionais e com a cultura organizacional que se pretende desenvolver.

\subsection{Papel estratégico da Comunicação Organizacional}

Nesse contexto, a Comunicação Organizacional apresenta um papel estratégico. A comunicação organizacional e a comunicação nas mídias sociais, conforme (Bueno, 2014), apresentam potencial para desempenhar papel importante na construção de capitais (o institucional e o de relacionamento) pois repercutem na formação da imagem, na reputação, na visibilidade das marcas, projeção de seus valores, criação e manutenção de 
relacionamentos. (Rossetti \& Morales, 2007) reconhecem que a comunicação associada à tecnologia da informação constitue mecanismo de suporte à integração do conhecimento e principal incorporador de valor em uma empresa. (Mourão \& Oliveira, 2016), (Pereira, 2014), (Baldissera, 2009), (Reis, 2004) e (Kunsch, 2003) defendem o papel estratégico da comunicação com foco na interação e compartilhamento do conhecimento. No entanto, segundo Mourão \& Oliveira (2016), a formação dos profissionais que atuam na área de comunicação ainda se apresenta sedimentada por uma base funcionalista do processo de comunicação, evidenciando o distanciamento entre teoria e prática. Como consequência, embora o encadeamento histórico-conceitual da comunicação caminhe em direção ao paradigma interacional, prevalece a hegemonia do clássico-informacional, que a relaciona à mera transmissão de informações, e não a ideia de compartilhamento. (Marchiori, 2008) afirma que não há mais como trabalhar apenas a estrutura técnica da comunicação dissociada da estratégia de pessoas. Ainda de acordo com Kunsch (2003), a comunicação é primordial para a compatibilização dos interesses dos empregados e da empresa, por meio do estímulo ao diálogo, à troca de informações e experiências e à participação de todos os níveis hierárquicos. É importante, como evidencia Pereira (2014), que a comunicação esteja incorporada à dinamização global da organização e orientada de forma continuada para a conquista de desafios constantes, superando as expectativas de todos os intervenientes. (Oliveira \& Lima, 2016) consideram que a parte fundamental do processo de mudança da cultura organizacional está na comunicação objetiva e eficiente, em que novos valores e conceitos são continuamente compartilhados pela organização, a fim de provocar a mudança de comportamentos e possibilitar a transformação cultural.

\section{Metodologia}

Este estudo consiste numa pesquisa qualitativa adotada como meio para exploração e entendimento do significado que os dirigentes atribuem às questões da cultura, da comunicação e da gestão da comunicação da Companhia pública em estudo (doravante denominada Companhia $Y$, para garantia de sigilo). O processo de pesquisa pode ser entendida como uma pesquisa social, usando a metodologia científica para conhecer o campo da realidade social tendo na observação uma das possíveis fontes de conhecimento (Gil, 2008). Como instrumento de coleta de dados, adotou-se entrevistas sobre temas do ambiente de trabalho dos dirigentes (Creswell, 2010) na forma de entrevista estruturada que, segundo Gil (2008), desenvolve-se a partir de questionamentos abertos considerando-se os objetivos do estudo e a revisão de literatura realizada contemplando uma breve introdução com informações gerais da Companhia Y e uma contextualização sobre o trabalho, subdividido em duas seções. A primeira, abrangeu a coleta de dados sobre o perfil e a vivência profissional dos entrevistados. A segunda consistiu de 25 questões relacionadas aos elementos culturais envolvidos na disseminação do conhecimento e ao papel e aos desafios da comunicação para a implantação de um sistema-piloto de gestão do conhecimento na Companhia Y. A análise de conteúdo foi a técnica adotada por abranger um conjunto de técnicas de análise de comunicações, ao gosto de diferentes opções teóricas, mas afinada com a natureza do problema de pesquisa e com a criatividade do pesquisador (Colbari, 2014). A análise permitiu o acesso ao conteúdo de um texto, aqui entendido de forma ampla, delimitando-se unidades de codificação e um sistema classificatório e objetivo, em que se identificou a frequência da presença (ou ausência) de itens de sentido (Bardin, 2011) e envolveu três fases: a) pré-análise, que compreendeu a triagem do material e a definição do método a ser utilizado; b) exploração do material; c) tratamento dos dados e interpretação, que representam, por sua vez, o desenvolvimento de deduções e resultados da pesquisa, podendo as hipóteses ser confirmadas ou não. As análises dos conteúdos das respostas aos questionamentos propostos nas entrevistas foram subdivididas em 3 agrupamentos, sendo: Conhecimento e a Gestão do Conhecimento; Cultura Organizacional e Comunicação Organizacional que, por sua vez, incluiu três subagrupamentos: i) a importância e o papel da comunicação num contexto de Gestão do Conhecimento; ii) ferramentas de comunicação já 
utilizadas pela Companhia $Y$ e, iii) instrumentos com potencial para trazer contribuições efetivas. As falas das entrevistas foram analisadas segundo os agrupamentos e subagrupamentos com vistas a identificar os maiores desafios a serem enfrentados referentes à cultura e à comunicação organizacionais, para a implantação de um sistema de gestão do conhecimento, na visão dos gestores de uma companhia pública brasileira.

\subsection{Contexto de estudo}

A Companhia Y é formada por 11 diretorias, 47 superintendências e 151 gerências. Foram selecionados dirigentes das áreas de Relacionamento e Recursos Humanos (quatro gerentes e um superintendente), de Comunicação (dois gerentes e um superintendente), de Tecnologia da Informação (três gerentes e um superintendente), da Saúde, Higiene e Segurança do Trabalho (um gerente), de Tecnologia e Inovação (três gerentes) e Relacionamento e Recursos Humanos (alta direção), totalizando 17 sujeitos. A opção por focar a visão dos dirigentes justifica-se na medida em que representa um grupo fundamental no processo de mudanças, sendo importante a sua participação e envolvimento em todas as etapas desse processo (Wood, 2009; López e Junquera, 2013). A Companhia $Y$ vem, ao longo dos anos, reduzindo seu quadro de pessoal, por meio de programas de incentivo à demissão, que tem ocorrido desde 1999. Nos anos de 2016 e 2017, com o Programa de Desligamento Voluntário Programado, em continuidade à política de desligamento de empregados em condições de aposentadoria, a redução alcançou quase 2000 pessoas. A terceirização, também, contribuiu para a redução de funcionários. A Companhia $Y$ apresenta uma demanda de modelo de gestão do conhecimento tecnológico que integre as diferentes fontes de conhecimento e utilize o potencial das tecnologias de informação e comunicação para mapear, registrar, comunicar, transferir e disseminar o patrimônio de conhecimento tecnológico da organização, sendo fundamental, neste contexto, cuidar da retenção do conhecimento crítico daqueles que saem.

\section{Análises e Resultados}

\subsection{Sobre o conhecimento e a gestão do conhecimento}

As análises demostram que a Companhia $Y$ enfrenta vários desafios, apesar de haver diversos procedimentos técnicos, normas de conduta e documentos armazenados em sistemas compartilhados pela intranet, que funcionam como um repositório de arquivos de uso comum. Identificou-se, no entanto, um gargalo na prática do compartilhamento de conhecimento de caráter tácito. A maioria dos entrevistados mencionou a carência desse compartilhamento nas interações entre as áreas, que possibilitaria a troca do conhecimento de valor agregado, por meio de discussões e intercâmbio de experiências. Conforme afirmam Davenport e Prusak (2003), a construção do conhecimento exige uma interação decorrente das relações sociais e das relações de trabalho entre os colaboradores de uma organização.

Nesta pesquisa foram entrevistados $8,13 \%$ do total de dirigentes da Companhia $Y$, correspondendo a 17 dirigentes. Os gráficos apresentados na figura 1 apresentam a distribuição desses 17 dirigentes segundo seus cargos (gerente, superintendente e alta direção), gênero (masculino-M, feminino - F) e formação profissional (administração, comunicação, engenharia, matemática e outra). 
Figura 1

Cargos, gêneros e formação dos dirigentes entrevistados.

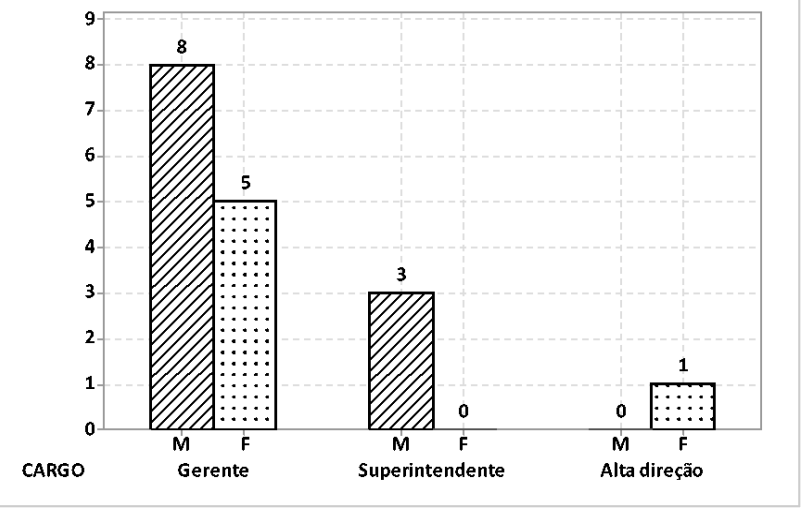

(a)

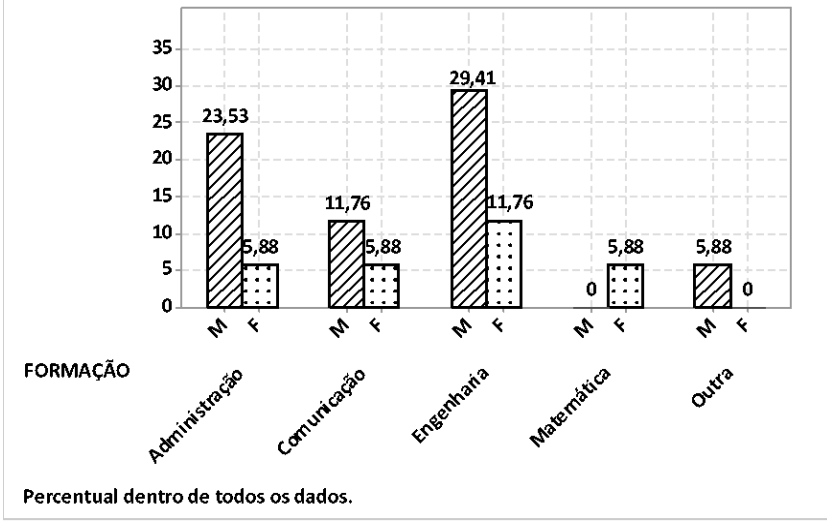

(b)

Fonte: Autoria própria.

Analisando a figura 1, observa-se no gráfico (a) que o maior percentual é de dirigentes de gênero masculino $(70,6 \%)$ que exercem as funções de gerente e superintendente enquanto um percentual menor $(29,4 \%)$ corresponde às dirigentes do gênero feminino que exercem as funções de gerente e alta direção. A formação profissional dos dirigentes apresentada no gráfico (b), apresenta os maiores percentuais para a área de exatas (Engenharia, sendo $29,41 \%$ do gênero masculino e $11,76 \%$ do gênero feminino), seguida de Administração (sendo $23,53 \%$ do gênero masculino e $5,88 \%$ do gênero feminino), Comunicação (sendo $11,76 \%$ do gênero masculino e $5,88 \%$ do gênero feminino) seguida de Matemática $(5,88 \%)$ e outra $(5,88 \%)$.

Pode-se inferir que as características de conservadorismo e a tendência de manter uma hierarquia rígida na cultura de gerenciamento do conhecimento poderiam estar relacionadas ao ambiente das profissões mais ligadas às áreas de exatas (engenharia e suas modalidades). A presença de representantes masculinos superior ao número de representantes femininas nos cargos de direção, com formação nas áreas de administração e engenharias, conforme o gráfico (b), poderia demonstrar uma tendência à manutenção de práticas mais conservadoras no ambiente de trabalho.

A análise dos relatos permite deduzir que o conhecimento e sua gestão se encontram mais na esfera prescritiva e menos na interativa, ou seja, conhecimentos padronizados, normatizados e de caráter mais explícito, racional e sistemático existem na Companhia e são, de fato, compartilhados. O conhecimento tácito, porém, não parece, ainda, dominante. A relação entre essa observação e o fato de que muito do conhecimento tem se perdido com a saída de empregados por meio de programas de desligamento, como o Programa de Desligamento Voluntário Programado, explica-se pelo fato de o conhecimento de valor agregado não ser estimulado ao compartilhamento. As falas seguintes demonstram essa perda: "Os que chegaram tiveram que achar seu caminho e faltou tempo de transição para absorverem o conhecimento tácito dos que saíram." (E1); "Não trabalham com uma Gestão do Conhecimento contínua - o que mais temos hoje é perda de conhecimento - o processo não é sustentado. Não tem como repor esse conhecimento. Se for uma saída natural, você consegue alternativas. Com o Programa de Desligamento Voluntário Programado temos saídas muito rápidas." (E 10).

Como ponto positivo, foi mencionada a presença de canais que multiplicam conhecimentos via treinamentos ministrados pelos empregados, além de outros voltados à transmissão do conhecimento técnico ao público operacional. Quanto à disseminação e ao compartilhamento do conhecimento, foram feitas críticas a alguns aspectos da gestão da Companhia Y que dificultam a difusão do conhecimento entre as áreas e os empregados. 
O modelo hierárquico rígido, como disseram os entrevistados, acentua a segregação entre liderança e liderados, constrangendo a transferência do conhecimento dos gestores às suas respectivas equipes. A cultura parece ser favorável somente para o conhecimento mais sistematizado e práticas de compartilhamento são apontadas como iniciativas individuais, ou seja, falta institucionalização. Isso resulta numa disseminação informal e em nichos: "A Companhia não incentiva a prática de compartilhar o conhecimento entre os empregados. Não existe um programa ou algo formatado, definido. Não está institucionalizado. Vai de cada um, de cada grupo. Vejo mais uma transferência de conhecimentos padronizados, prescritos e menos de atividades reais." (E 7).

Apesar de alguns entrevistados salientarem que a Companhia $Y$ incentiva os empregados ao compartilhamento do conhecimento, foram descritos problemas identificados pela liderança, como a questão de o conhecimento ser mais individualizado e esse traço de personificação faz com que o indivíduo entenda que o conhecimento é dele e não da Empresa. Segundo os relatos, os empregados que têm mais tempo de casa, por exemplo, associam informação à poder, o que em nada facilita que o compartilhem. Soma-se a isso a questão do conflito de gerações, salientado por diversos entrevistados, segundo os quais os empregados que entraram recentemente na Empresa não necessariamente conseguiram absorver o conhecimento tácito dos que saíram: "O conhecimento hoje é visto de uma forma personificada. O conhecimento na organização é da pessoa, não é da Empresa. A pessoa que carrega esse conhecimento vira referência no assunto e passa a ser proprietária desse conhecimento. Esse conhecimento, porém, não é mapeado e registrado na maioria dos casos. Esse conhecimento é levado quando a pessoa sai da Empresa." (E 16); "Há duas gerações conflitantes aqui. O conflito de geração não está resolvido na Empresa. Enquanto esse conflito não for resolvido, não vai existir ferramenta de Gestão do Conhecimento que seja efetiva." (E 14).

Apresentam-se, na figura 2 dados sobre a faixa etária dos dirigentes e dos participantes das equipes.

Figura 2

Faixa etária do capital humano

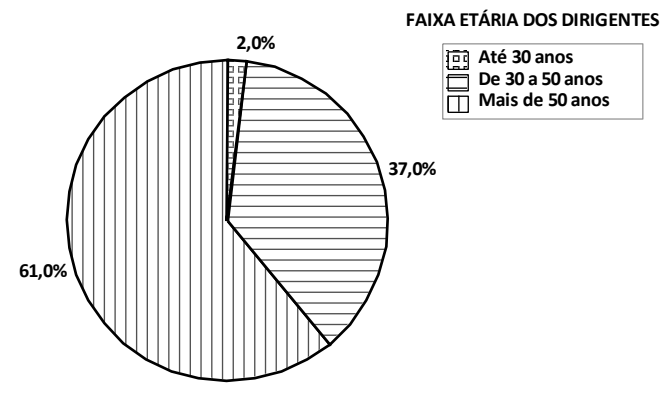

(a)

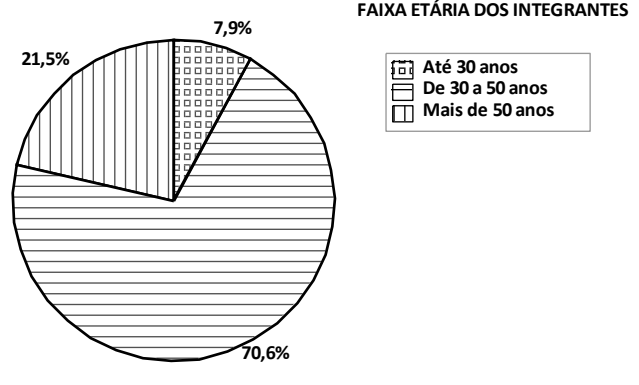

(b)

Fonte: Autoria própria.

A análise da figura 2 permite identificar no gráfico (a) que, dos dirigentes da Companhia $Y$ entrevistados, apenas $2 \%$ estão abaixo de 30 anos, $37 \%$ estão na faixa de 31 a 50 anos e a maioria com $61 \%$ estão acima de 50 anos. Conforme o gráfico (b), por outro lado, 7,9\% dos integrantes das equipes estão abaixo de 30 anos, 21,5\% apresentam mais de 50 anos e a maioria com 70,6\% estão na faixa de 30 à 50 anos. 
Também foi identificado que existe uma falta de reconhecimento e incentivo da Companhia $\mathrm{Y}$ ao compartilhamento do conhecimento, associada à escassez de apoio da liderança. Nesse sentido, (Gnecco Júnior, Pereira, Santana, \& Tecchio, 2010) apontam que os possíveis fatores críticos de sucesso para a Gestão do Conhecimento seriam: o papel da liderança, seu comprometimento e apoio; medição de desempenho; iniciativas em tecnologia que possam apoiar a implantação de estratégias da Gestão do Conhecimento; suporte de especialistas; cultura amigável e processos, políticas e atividades que regem a organização. Nesse caso, vê-se que todos esses fatores não parecem presentes na Empresa em estudo.

\subsection{Sobre a cultura organizacional}

$\mathrm{O}$ segundo agrupamento envolve os relatos que mencionam questões referentes à cultura da Companhia $\mathrm{Y}$, sobretudo no que tange à liderança e ao processo de mudança organizacional pelo qual ela passa. Todos os relatos dos entrevistados caracterizaram a cultura da Empresa como forte, hierárquica, rígida e conservadora, como atesta a seguinte fala: "A Empresa tem uma cultura muito forte. Do ponto de vista empresarial faz uma empresa cadenciada, que corre pouco risco, tem um sistema de tomada de decisão lento, travado, quartel. A cultura é travada, restringe muito as pessoas." (E 13).

Conforme o estudo de (Veiga \& Machado, 2011), a cultura da Empresa em estudo ainda aparece como aquela que pratica a estabilidade e a previsibilidade. Um elemento valorizado como símbolo de poder e autoridade nesse tipo de cultura é o status, manifesto em símbolos mencionados por um entrevistado (elevadores exclusivos, formas distintas de se vestir etc). Nesse modelo bem próprio, foram identificadas vertentes que permitem esclarecer os desafios com os quais a Gestão do Conhecimento e sua disseminação se deparam diante de aspectos intrínsecos à cultura. Dentre os fatores mais relevantes identificados, destacam-se a estrutura complexa, verticalizada e inchada, o que torna a tomada de decisão mais lenta e limitada. Segundo os participantes da pesquisa, a cultura restringe as pessoas, fracionando as áreas que pouco se comunicam. Parece que a cultura apresenta pouca flexibilidade para mudanças e o corporativismo é forte em todos os segmentos, sendo cada qual com seu interesse. A mudança constante da alta direção promove a renovação de, pelo menos, $50 \%$ do quadro, o que impacta diretamente a Gestão do Conhecimento. Ademais, a Lei n. 8.866, que rege as empresas públicas, por meio de licitações e concorrências, acarreta perda na agilidade dos processos, impactando a cultura. Mas os depoimentos sinalizam que a Companhia Y passa por um processo de mudança, fruto de todas essas alterações que têm ocorrido na própria organização, tais como: "Cultura forte no ponto de vista de ter uma linguagem técnica específica e de certa forma de sucesso. É uma empresa de referência no setor (...). Conservadora e hierárquica, mas já foi pior." (E 9).

A expressão já foi pior, em especial, retrata que as transformações que estão ocorrendo parecem benéficas ao entrevistado, talvez porque devem favorecer uma cultura mais aberta ao diálogo, portanto, mais propícia à Gestão do Conhecimento. A predominância da identificação da cultura da Empresa como forte remete à afirmação de Freitas (1997), que conclui que uma cultura forte é boa quando favorece a integração e ruim quando pode ser tomada como freio para a incorporação de processos de mudança. Em especial num contexto como o que se apresenta, em que a Empresa manifesta a necessidade de reter o conhecimento que está se perdendo diante de um mercado mais dinâmico e competitivo, grandes mudanças culturais parecem favoráveis, porém improváveis e, nesse sentido, essa é uma preocupação que a Organização deve ter. Ainda relacionado a esse aspecto, Freitas (2007) afirma que a cultura organizacional pode ser entendida como uma metáfora ou uma variável. Na última perspectiva, ou aquela que este estudo adota, é possível manipulá-la ou gerenciá-la conforme os interesses organizacionais, ou seja, trata-se de uma visão instrumental do assunto que enxerga a possibilidade de alterar as características culturais da Empresa. No entanto, mesmo para autores dessa área, não se trata de uma tarefa de fácil execução, o que é corroborado pelos depoimentos anteriormente analisados. (Cota, Ituassu, 
\& Vasconcelos, 2016) analisando o comprometimento organizacional em área técnica de banco público brasileiro concluem que a internalização de valores organizacionais trata-se de um importante ativo de uma empresa. $\mathrm{Na}$ visão ampla de Schein e Schein (2017), quando a organização tem um propósito, uma nova estratégia, um problema para ser resolvido, ou uma agenda de mudança, determinar como a cultura afeta a questão não é apenas útil, mas necessária, na maioria dos casos. Em contraste à esses desafios, os entrevistados apontaram elementos positivos da cultura da Companhia que poderiam apoiar a implantação de um sistema de Gestão do Conhecimento. Segundo os entrevistados, os empregados gostam de trabalhar na Empresa e, mesmo diante das dificuldades, eles se empenham e se dedicam para tentar conseguir reverter os problemas com que se defrontam. Os papéis de cada área são claros e bem definidos, o que facilita que trabalhem em prol do crescimento da Companhia Y. Além disso, seu corpo técnico e sua competência são referências no mercado, tornando-os, ainda, mais fortes e comprometidos.

Para alguns entrevistados a Companhia $Y$ é transparente, tem mudado muito e se adaptado bem às transformações e apresenta um perfil menos estatal e mais arrojado, atento ao mercado, buscando foco em resultados. A entrada de novas pessoas também é um fator positivo para trazer novas ideias à Empresa. Grande parte dos líderes entrevistados citou que o capital humano é um dos aspectos mais favoráveis da cultura da empresa, reconhecendo que o seu envolvimento e o sentimento de orgulho e de pertencimento à empresa possibilitam uma melhor abertura para mudanças e melhorias.

Apresenta-se na figura 3 dados sobre o tempo dos dirigentes entrevistados na condução dos cargos a que foram designados e o número de integrantes nas equipes por eles lideradas.

Figura 3

Tempo dos dirigentes na condução dos cargos

e número de integrantes nas equipes

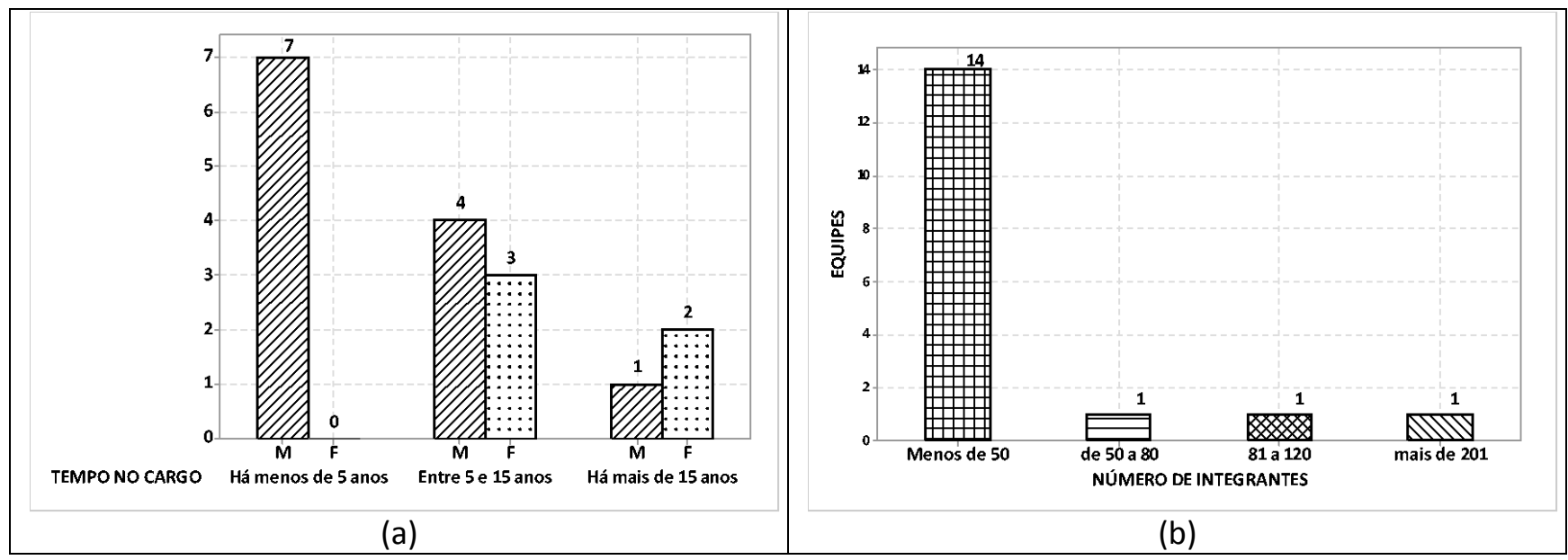

Fonte: Autoria própria.

Observa-se no gráfico da figura 3 (a) que 07 participantes do estudo $(41,18 \%)$ estão há menos de cinco anos na Companhia $Y$, indicando um movimento recente de renovação na organização, enquanto outros 07 (portanto, 0 mesmo percentual de 41,18\%) já se encontram entre 5 a 15 anos e somente 03 (17,64\%) ocupam os cargos há mais de 15 anos. Analisando a figura 3 (b) observa-se que existe uma grande diversidade nos tamanhos das equipes, sendo que as equipes de 14 dirigentes (82,35\%) apresentam menos de 50 integrantes, sendo um fator que pode facilitar, mas não determinar, a integração entre os membros delas participantes. 
Ao comparar os aspectos positivos e negativos identificados nas respostas dos diferentes dirigentes, pode-se reconhecer a probabilidade de existência, na mesma organização, de diversas culturas diferentes, como mencionado em Schein $(2004$, p. 9) que afirma "[...] a cultura é um fenômeno multidimensional, multifacetado, dificilmente redutível a algumas dimensões principais." Essas várias culturas também se tornam visíveis em expressões como foi citado, entre os entrevistados, que é preciso trabalhar mais a parte cultural da liderança, já que ela se encontra orientada para resultados. Assim, o fator técnico vem à frente do cultural, sem que se perceba que este último é igualmente importante para o desempenho da Empresa, como identificado nas seguintes falas: "O relacionamento entre os gestores de área é formal. Embora haja discussões, não há um ambiente criado que traga oportunidades; não há espaço de compartilhamento e disseminação do conhecimento entre os gerentes. (E 5); "As melhoras que os gestores procuram são sempre eficiência operacional e produtividade. As áreas estão muito focadas no operacional, serviço de bombeiro ('apagar o fogo') - não existe muita oportunidade para que os gerentes possam trabalhar mais no aspecto estratégico." (E 4).

Contudo, este não é um problema único da Companhia Y em estudo. (Rigo, Souza Filho, \& Souza, 2007) reforçam a incompreensão, por parte das empresas, do papel da gestão de pessoas em processos de implantação de programas de Gestão do Conhecimento. Outro aspecto da liderança, observado pelos entrevistados, diz respeito à condução dos procedimentos internos, que funcionam somente com o apoio da diretoria. López e Junquera (2013) afirmam que a transferência do conhecimento tácito necessita de maior interação entre os indivíduos, porém identifica-se que há pouca autonomia entre gerentes e superintendentes para assumirem mais responsabilidades, limitando-se à execução de tarefas menos estratégicas. A diretoria funciona como uma cultura própria, caracterizada pela centralização do poder, fato mencionado em diversos relatos, dentre eles, o seguinte: "Superintendentes e gerentes poderiam ter mais autonomia e tomar decisões rápidas que não dependessem tanto da diretoria, que poderia cuidar somente de assuntos extremamente estratégicos. A centralização de poder é incompatível com o porte da empresa." (E 12).

Vale ressaltar o argumento de (Ghoshal \& Tanure, 2004), para quem a alta direção influencia a mudança de comportamento, de aceitação de algo novo, por meio de sua própria mudança, destacando-se a importância da liderança no processo de implantação de um sistema de Gestão do Conhecimento. (Albertin , 1995) assinala o suporte do corpo executivo, envolvendo sua participação, envolvimento, estilo gerencial, fornecimento e recepção de informações e apoio como um dos mais importantes fatores críticos de sucesso nesses processos. Como identificado em Schein (1992), a cultura e a liderança têm, assim, forte relação e relevante papel, como identificado na seguinte fala: "A liderança é um catalisador. Tem o papel de exemplo para os demais. A liderança tem que tomar a frente em relação a qualquer projeto que se formate em relação a isso." (E 7).

No que tange à necessidade de inserir novas tecnologias na implantação do sistema de Gestão do Conhecimento, foi assinalado pelos entrevistados que alguns líderes apresentam uma resistência maior em relação ao uso de novas ferramentas e tecnologias. Percebeu-se também que os gestores que têm maior facilidade com tecnologia obtêm melhores resultados, como destacado na fala seguinte: "Os gerentes que têm facilidade com tecnologia se desenvolvem mais rápido. É preciso expandir essas melhores práticas para mais de uma área." (E 1).

Bhatt (2002), ao abordar a resistência perante a implantação de um sistema de informação, afirma que, apesar de os gestores concordarem com a implantação de novas tecnologias e modelos organizacionais para gerir o conhecimento, as ações não são facilmente adotadas, especialmente, pela fidelidade às maneiras tradicionais de administrar o capital estrutural físico com o auxílio de procedimentos e normas organizacionais. Unindo o que diz esse autor ao que afirmam outros, sobre o papel da liderança (Schein, 1992; Albertin, 1995; Gnecco Júnior et al., 2010), torna-se importante ressaltar que cabe aos líderes aderir às tecnologias que a Empresa passa a utilizar, para dar o exemplo e o incentivo pelo modelo. 


\subsection{Sobre a Comunicação Organizacional}

A análise de diversos relatos demonstra que a comunicação organizacional é um elemento forte de formação de cultura, essencial para o engajamento e a motivação dos empregados. Alguns entrevistados defendem que, além de ser o instrumento para o estabelecimento das relações entre as pessoas, seria também a principal motivadora da implantação de um sistema de Gestão do Conhecimento. Outros apoiam a ideia de que a comunicação seria a última etapa do processo e que haveria várias fases anteriores que precisariam estar bem estruturadas. Outros entrevistados afirmam que veículos de comunicação já utilizados internamente e as campanhas que a Empresa realiza poderiam apoiar a disseminação dessa cultura de compartilhamento. Uma das respostas trouxe uma perspectiva aliada ao paradigma interacional da comunicação (Mourão e Oliveira, 2016) defendendo que a mesma ultrapasse a função de informar: "Tem que ter um trabalho de convencer as pessoas de que aquilo é bom. As pessoas têm um processo de convencimento. Mais do que um processo de divulgação[...]."(E 13).

Como precedente da ação, a comunicação, sob essa visão, precisaria demonstrar e convencer as pessoas de que a implantação de um sistema de Gestão do Conhecimento seria benéfica para a Empresa e o próprio funcionário, envolvendo-o de modo que partilhasse das informações, não só as conhecendo, mas acreditando nelas, como mencionado nas falas seguintes: "A comunicação tem que mostrar que o sistema vai agregar valor. Adesão num primeiro momento e, além da comunicação, cabe ao líder incentivar." (E 10); "Ter um plano de comunicação bem abrangente que conseguisse chegar a todos os empregados. Algo constante e que colocasse os principais colaboradores como personagens da ação, criando uma ligação afetiva, (...)." (E 12).

Sob esse aspecto, Baldissera (2009) afirma que a comunicação não se limita somente aos processos formais, da fala organizada, que engloba o conteúdo que a organização seleciona de sua identidade e, por esferas comunicacionais, precisa envolver crenças e levar em conta medos, anseios e expectativas. Nessa mesma ótica, Kunsch (2003) argumenta que a comunicação organizacional se tornou um dos mais importantes elementos para a estratégia do negócio. Para a autora, ela é primordial para a consonância dos interesses dos empregados e da Empresa, por meio do estímulo ao diálogo, à troca de informações e experiências e à participação de todos os níveis hierárquicos. Apesar de uma evolução gradativa dos modelos mecânicos da transmissão unilateral de informações para a implantação de processos comunicativos mais interativos, ainda falta, em grande parte das empresas, uma comunicação que permita a abertura de canais dialógicos de fato, em que as pessoas sejam consideradas em primeiro lugar (Kunsch, 2012). Isso fica visível nos depoimentos obtidos: para os entrevistados, está claro que, à comunicação organização, não basta informar. É preciso colocar os funcionários como protagonistas desse processo, agentes diretamente implicados na mudança. Cabe falar e ouvir, dar espaço para inquietações e sugestões e elaborar estratégias levando em conta o que sentem e pensam. É possível perceber, na maioria das respostas, que a comunicação é reconhecida como componente essencial na disseminação da importância do sistema de Gestão do Conhecimento, responsável também pelos seus benefícios e resultados, esclarecendo, por meio de uma linguagem acessível, direta e transparente, o propósito do projeto. As seguintes falas atestam essa percepção: “A comunicação pode contribuir para o sucesso de uma Gestão do Conhecimento. Engajar os empregados e gestores, motivá-los e deixar isso bem claro - qual o benefício disso para empresa e pessoas." (E 15); "A comunicação deveria ser a principal alavancadora. É ela que permeia as pessoas, aumentaria a liga entre as pessoas, abertura para perceber quais ferramentas de fora para dentro promoveriam a transformação cultural." (E 6).

Os depoimentos corroboram, portanto, o que a literatura afirma sobre a relevância da comunicação clara e objetiva dos objetivos e das mudanças a serem implementadas, nesse processo (Wood Jr., 2009). Nas respostas 
coletadas também ficou clara a relevância do incentivo do líder, sobretudo por meio da comunicação face a face, que é a mais efetiva, tais como: "A comunicação é essencial, processo desenhado, como vai levar isso aos públicos, linguagem acessível que seja clara, transparente, direta e que traga propósito e confiança. $\mathrm{O}$ mais importante é que a liderança pratique isso." (E 2).

Nesse âmbito, Marchiori (2008) defende que a cultura do diálogo cria conexões que se concretizam em comportamentos positivos e proativos. Schein (1992) reforça isso, afirmando que a liderança é o grande impulsionador da cultura e realça que ela é originalmente a fonte de crenças e valores que definem como os membros da organização se comportam. Sem uma comunicação efetiva, portanto, isso não seria possível.

Dentre as ferramentas de comunicação que existem na Companhia $Y$ em estudo, listadas pelos entrevistados, destacam-se: intranets, o jornal diário para informações diversas, e-mails, cartazes e jornal mural, revista, grupos de WhatsApp das áreas e comunicação face à face. Apesar de as organizações divulgarem suas intranets como espaços relacionais, os empregados as percebem e as utilizam como ambientes informais. A cultura dessas organizações e a visão de alguns gestores não estimulam o tempo gasto nesse tipo de atividade, ao considerá-la supérflua. Em relação à Gestão do Conhecimento, foram identificados os seguintes instrumentos: o Visão e Ação (boletim mensal); o Wiki, que funciona como um repositório de conhecimento, porém, é subutilizado. Segundo os entrevistados, falta cultura de uso, como a seguir mencionado: "Nós temos um banco de dados com quase três mil linhas, pronto para ser usado em formato Wiki (dentro da intranet), mas não conseguimos utilizar. Se for atualizado, teríamos uma ferramenta poderosíssima na mão [...]." (E 12) ; "Outra ferramenta são os ambientes SAP - Solution Manager (...), que armazena toda documentação funcional dos processos que são tocados no sistema. Para os demais ambientes garante um pouco do conhecimento do mundo do $\mathrm{TI}$, mas não atende a Casa." (E 10).

Todos os entrevistados apresentaram sugestões de melhorias em relação à disseminação do conhecimento, da cultura e da comunicação, sendo coletadas mais de 60 ideias, dentre as quais, a migração de comunicação linear/informacional para a participativa e interativa, mas que ainda precisa evoluir principalmente em função da cultura da Empresa.

\section{Conclusões}

Este estudo objetivou analisar os maiores desafios a serem enfrentados no que se refere à cultura e à comunicação organizacionais para a implantação de um sistema de Gestão do Conhecimento, na visão de gestores da Companhia Y em estudo. Para alcançá-lo, foram entrevistados 17 dirigentes/gestores, cujos relatos foram submetidos à análise de conteúdo. Focalizando o primeiro agrupamento analisado, Conhecimento e Gestão do Conhecimento, identificou-se que os programas de demissão voluntária adotados nos últimos anos fizeram com que muito do conhecimento retido pelos funcionários mais experientes fosse perdido pelo fato de a cultura não incentivar a transmissão do conhecimento tácito. Outro entrave à disseminação do conhecimento refere-se à associação entre detenção do conhecimento e fonte de poder. No segundo agrupamento analisado, Cultura Organizacional, identificou-se que as características predominantes na cultura da Companhia $Y$ são o conservadorismo, a hierarquia rígida, o isolamento da diretoria e o conflito de gerações os quais, para os participantes da pesquisa, são os principais inibidores para a concepção de uma mudança organizacional. Por outro lado, apesar dos desafios, foram identificados elementos favoráveis da cultura da Companhia Y que podem propiciar uma abertura à mudança, tais como o engajamento do seu capital humano e o domínio do conhecimento técnico dos empregados, que são, inclusive, referência no mercado. No terceiro agrupamento investigado, a Comunicação Organizacional, identificou-se na maioria das respostas que a comunicação é reconhecida como componente essencial na disseminação da importância do sistema de Gestão do 
Conhecimento, responsável também pelos seus benefícios e resultados, porém, na prática observa-se que as redes internas (intranets) não são adequadamente utilizadas pelos empregados como espaços relacionais mas como ambientes informais enquanto na visão de alguns gestores o tempo dispensado nas intranets seria supérfluo. Outros instrumentos de comunicação, como os boletins mensais (Visão e Ação) e o portal da Companhia $Y$, que poderia ser um wikipedia, é subutilizado, não funciona como um banco de dados, está desatualizado e não há investimento para reverter esse processo. Segundo os entrevistados, os mecanismos de comunicação dentro da empresa apresentam característica linear/informacional, não participativa e não interativa, em função da cultura da própria Companhia Y. Nas entrevistas, foi salientada a necessidade de o líder ser um agente transformador, influenciador e com competência para alavancar a equipe. Espera-se que os resultados desta pesquisa melhorem a compreensão sobre a relevância da cultura organizacional considerando que esta envolve pessoas e, portanto, deve enfatizar os aspectos relacionados às suas crenças, normas, valores e premissas. Da mesma forma, compreender o papel que a comunicação desempenha nesse contexto é importante, dado que poucos estudos se dedicam ao tema, essencial para a adesão dos funcionários às inovações propostas. Pesquisas futuras podem descrever o processo de implantação de sistemas de Gestão do Conhecimento em organizações do setor público que obtiveram bons resultados e os maiores problemas enfrentados nesse processo, assim como investigar a perspectiva dos liderados a respeito desse processo.

\section{Referências}

Albertin , L. A. (1995). Administração de informática e a organização. Revista de Administração de Empresas, 34.

Alvarenga Neto, R. C. (2005). Gestão do conhecimento em organizações: proposta de mapeamento conceitual integrativo. São Paulo: Saraiva.

Andrade, A. R., \& Roseira, C. A. (2018). A informação como elemento de integração entre propósito, processos e pessoas: um estudo em instituições portuguesas. Gest. Prod., 25, pp. 107-116. doi:http://dx.doi.org/10.1590/0104-530X2642-16.

Baldissera, R. (2009). Comunicação organizacional na perspectiva da complexidade. Revista Brasileira Comunicação Organizacional e Relações Públicas, 6.

Bardin, L. (2011). Análise de conteúdo. (L. A. Reto, \& A. Pinheiro, Trads.) São Paulo: Edições 70.

Bhatt, G. D. (2002). Management strategies for individual knowledge and organizational knowledge. Journal of Knowledge Management, 6.

Bueno, W. C. (2014). Avaliando os ativos intangíveis das organizações nas mídias sociais: os limites das métricas. XXIII Encontro Anual da Compós. Belém.

Carvalho, F. A. (2012). Gestão do Conhecimento. São Paulo: Pearson.

Choo, C. H. (2003). A organização do conhecimento: como as organizações usam a informação para criar significado, construir conhecimento e tomar decisões. São Paulo: SENAC.

Colbari, A. (2014). A análise de conteúdo e a pesquisa empírica qualitativa. Vitória: Editora da UFES.

Contador, J. L. (2005). Gerenciamento de projetos com PERT/CPM. In: Gestão de operações. São Paulo: Edgard Blücher. 
Cota, F., Ituassu, C. T., \& Vasconcelos, F. W. (2016). Comprometimento organizacional numa área técnica de um Banco Público Brasileiro. Espacios. Vol. 37 (№ 20).

Creswell, J. W. (2010). Projeto de pesquisa: métodos qualitativo, quantitativo e misto (3a ed.). (M. F. Lopes, Trad.) Porto Alegre: Artmed.

Cruz, S. G., \& Ferreira, M. F. (2015). Perception of organizational culture and knowledge management in hospitals using different management models. Referência, 4.

Davenport, T. H., \& Prusak, L. (2003). Conhecimento empresarial: como as organizações gerenciam o seu capital intelectual. Rio de Janeiro: Campus.

Dazzi, M. S., \& Angeloni, M. T. (2009). Compreendendo o significado de gestão do conhecimento e a importância da comunicação em seu compartilhamento - um estudo de caso. Revista Eletrônica do Mestrado Profissional em Administração da Universidade Potiguar.

Drucker, P. F. (1995). Managing in a time of great change. New York: Butterworth-Heinemann.

Fernandes, A. L., Alturas, B. B., \& Laureano, R. S. (2015). O papel da cultura organizacional na dinâmica de relacionamento entre as tecnologias da informação e a aprendizagem organizacional: um ensaio teórico voltado para o segmento da hotelaria. . Anais ANANPAD. Belo Horizonte.

Freitas, M. E. (1997). Cultura Organizacional: o doce controle no clube dos raros. In: Cultura organizacional e cultura brasileira. São Paulo: Atlas.

Freitas, M. E. (2006). Cultura organizacional: identidade, sedução e carisma. (5a ed.). Rio de Janeiro: FGV.

Freitas, M. E. (2007). Cultura organizacional: evolução e crítica. São Paulo: Thompson Learning.

Ghoshal, S., \& Tanure, B. (2004). Estratégia e gestão empresarial: construindo empresas brasileiras de sucesso: estudos de casos. São Paulo: Campus.

Gil, A. C. (2008). Métodos e técnicas de pesquisa social. . São Paulo: Atlas.

Gnecco Júnior, L., Pereira, M. F., Santana, J. Q., \& Tecchio, E. L. (2010). Gestão do conhecimento: fatores críticos de sucesso. Reuna.

Kunsch, M. K. (2003). Planejamento de relações públicas na comunicação integrada. São Paulo: Summus.

Kunsch, M. K. (2012). As dimensões humanas, instrumental e estratégica da comunicação organizacional: recorte de um estudo aplicado ao segmento corporativo. Revista Brasileira de Ciências de Comunicação.

López, S. P., \& Junquera, B. (2013). The relation between IT competency and knowledge management processes and its mediators. Tourism \& Management Studies, 9.

Marchiori, M. (2008). Cultura e comunicação organizacional: um olhar estratégico sobre a organização (2a ed.). São Caetano: Difusão.

Moreno, V., \& Santos, L. A. (2012). Gestão do conhecimento e redesenho de processos de negócio: proposta de uma metodologia integrada. . Revista Perspectivas em Ciência da Informação, 17.

Mourão , I., \& Oliveira, I. L. (2016). Comunicação organizacional: análise dos construtos teóricos e a práxis na formação do discurso. Conexão - Comunicação e Cultura, 15. 
Neves, F. S., \& Castro, J. M. (2010). A participação e o envolvimento dos servidores como fatores críticos para a implementação de um processo de mudança organizacional: um estudo de caso no governo do Estado de Minas Gerais. Encontro de Administração Pública e Governança.

Nonaka, I. (1994). A dynamic theory of organization knowledge creation. . Organization Science, 5.

Nonaka, I., \& Takeuchi, H. (1997). Criação do conhecimento na empresa. Rio de Janeiro: Campus.

Oliveira, R. R., \& Lima, J. B. (2016). Reflexão sobre a relação entre a mudança de cultura organizacional e a gestão do conhecimento. Perspectivas em Gestão \& Conhecimento, 6.

Paiva, K. M., \& Alves, G. B. (2013). Comunicação organizacional: meta-estudo com pesquisas brasileiras. Tourism\& Management Studies.

Pereira, M. S. (2014). Comunicação estratégica no contexto organizacional. Revista Internacional de Ciências, 4.

Perides, M. N., Vilela, N. S., Souza, C. B., Dutra, J. S., \& Casado, T. (2020). Cultura organizacional e gestão por competências: Caso GERDAU.

Reis, M. C. (2004). Comunicação e mudança organizacional: uma interlocução instrumental e constitutiva. Revista Brasileira de Comunicação Organizacional e Relações Públicas, 1.

Rigo, A. S., Souza Filho, N. A., \& Souza, D. C. (2007). Aspectos críticos da gestão do conhecimento (das pessoas) para a gestão de pessoas. Encontro de Gestão de Pessoas e Relações de Trabalho. Natal.

Rossetti, A. G., \& Morales, A. T. (jan./ abr de 2007). O papel da tecnologia da informação na gestão do conhecimento. . Ci. Inf., 36, pp. 124-135.

Runte, G. B., \& OLiveira, F. B. (2012). Gestão do connhecimento: os desafios da implantação de um modelo integrado: o caso ANS. Encontro de Estudos Organizacionais da ANPAD. Curitiba.

Sales, V. V., Ferreira, M. T., Paiva, R. C., \& Ziviani, F. (jan./abr. de 2019). A gestão de ideias no âmbito da gestão do conhecimento: catalisando a inovação nas organizações. Ci.Inf., 48, pp. 41-60.

Schein, E. H. (1992). Como preparar o terreno para a mudança na cultura organizacional. In: Organizational culture and leadership. São Francisco: Jossey-Bass.

Schein, E. H. (2004). Organizational culture and leadership (3rd ed.). John Wiley \&bSons.

Schein, E. H., \& Schein, P. (2017). Organizational culture and leadership (5th ed.). Wiley.

Silva, J. G., \& Vergara, S. C. (2003). Sentimentos, subjetividade e supostas resistências à mudança organizacional. Administração de Empresas.

Silveira, M. S., Ziviani, F., Ferreira, M. T., \& Paiva, R. C. (2018). Gestão de processo de compartilhamento do conhecimento tecnológico em uma empresa do setor elétrico (CEMIG). Inf. Inf. , 23. doi:10.5433/1981$8920.2018 v 23 n 2 p 538$

Toffler, A. (1990). Power shift knowledge, wealth and violence at the edge of the 21st century. New York: Bantam Books.

Veiga, S., \& Machado, M. M. (2011). Inter-relações entre forças influenciadoras e cultura organizacional. Psicologia em Revista, 17. 
Wood Júnior, T. (2009). Mudança organizacional (5a ed.). São Paulo: Atlas.

Xavier, L. P., Oliveira, M., \& Teixeira, E. K. (2012). Teorias utilizadas nas investigações sobre gestão do conhecimento. Risti - Revista Ibérica de Sistemas e Tecnologia da Informação.

Zandi, S., Moghadam, S., \& Ali Pour, A. (2016). The impact of organizational culture, IT and knowledge management on the organizational innovation. International Journal of Scientific Management \& Development.

Esta obra está bajo una Licencia Creative Commons Attribución-NoCommercial 4.0 International

(c) BY-NC 